\title{
Validity and reliability of a Malay version of the brief illness perception questionnaire for patients with type 2 diabetes mellitus
}

Boon-How Chew ${ }^{1,2^{*}}$ D, Rimke C. Vos ${ }^{2}$, Monique Heijmans ${ }^{3}$, Sazlina Shariff-Ghazali ${ }^{1}$, Aaron Fernandez ${ }^{4}$ and Guy E. H. M. Rutten²

\begin{abstract}
Background: Illness perceptions involve the personal beliefs that patients have about their illness and may influence health behaviours considerably. Since an instrument to measure these perceptions for Malay population in Malaysia is lacking, we translated and examined the psychometric properties of the Malay version of the Brief Illness Perception Questionnaire (MBIPQ) in adult patients with type 2 diabetes mellitus.
\end{abstract}

Methods: The MBIPQ has nine items, all use a 0-10 response scale, except the ninth item about causal factors, which is an open-ended item. A standard procedure was used to translate and adapt the English BIPQ into Malay language. Construct validity was examined comparing item scores and scores on the Diabetes Management Self-Efficacy Scale, the Morisky Medication Adherence Scale, the World Health Organization Quality of Life-brief, the 9-item Patient Health Questionnaire, the 17-item Diabetes Distress Scale, HbA1c and the presence of complications. In addition, 2-week and 4-week test-retest reliability were studied.

Results: A total of 312 patients completed the MBIPQ. Out of this, 97 and 215 patients completed the 2- or 4-weeks test-retest reliability questionnaire, respectively. Moderate inter-items correlations were observed between illness perception dimensions ( $r=-0.31$ to 0.53). MBIPQ items showed the expected correlations with self-efficacy $(r=0.35)$, medication adherence $(r=0.29)$, quality of life $(r=-0.17$ to 0.31$)$ and depressive symptoms $(r=-0.18$ to 0.21$)$. People with severe diabetes-related distress also were more concern (t-test $=4.01, p<0.001$ ) and experienced lower personal control (t-test $=2.07, p=0.031$ ). People with any diabetes-related complication perceived the consequences as more serious (t-test $=2.04, p=0.044$ ). The 2 -week and 4-week test-retest reliabilities varied between ICC agreement 0.39 to 0.70 and 0.58 to 0.78 , respectively.

Conclusions: The psychometric properties of items in the MBIPQ are moderate. The MBIPQ showed good cross-cultural validity and moderate construct validity. Test-retest reliability was moderate. Despite the moderate psychometric properties, the MBIPQ may be useful in clinical practice as it is a useful instrument to elicit and communicate on patient's personal thoughts and feelings. Future research is needed to establish its responsiveness and predictive validity.

Trial registration: ClinicalTrials.gov NCT02730754 registered on March 29, 2016; NCT02730078 registered on March 29, 2016.

Keywords: Beliefs, Brief illness perception questionnaire, Validity, Illness perceptions, Type 2 diabetes mellitus, Self-efficacy, Medication adherence, Quality of life, Emotional distress, HbA1c

\footnotetext{
* Correspondence: chewboonhow@gmail.com; chewboonhow@upm.edu.my

'Department of Family Medicine, Faculty of Medicine and Health Sciences,

Universiti Putra Malaysia, 43400 Serdang, Selangor, Malaysia

2Julius Center for Health Sciences and Primary Care, Department of General

Practice, University Medical Center Utrecht, Huispost Str.6.131, P.O. Box

85500, 3508, GA, Utrecht, The Netherlands

Full list of author information is available at the end of the article
} 


\section{Background}

Illness perceptions refer to the personal beliefs that patients have about their illnesses [1,2]. Among others, illness perceptions constitute beliefs about the typical complaints that belong to the illness, beliefs about the course, the consequences and the extent to which a disease is controllable either by self-care or medical care; they also include the understanding a patient has of the illness [2]. Illness perceptions evaluate the emotional impact of the illness directly and indirectly from the symptoms experienced and concern about the illness's consequences [3]. Illness perceptions are an important determinant of health behaviours such as in treatment adherence and via health behaviour they indirectly influence outcomes such as quality of life, functional recovery and clinical parameters [4]. Intervention studies have shown that illness perceptions can be changed [2, 5].

In both type 1 and type 2 diabetes mellitus (T2DM) patients illness perceptions have an independent effect on dietary management, physical exercise, self-monitoring of blood glucose, medication adherence, foot care, smoking cessation, appointment attendance, anxiety and depression [6]. Croatian adults with T2DM who reported a better illness understanding and experienced more personal control had a healthier lifestyle than those with more negative illness perceptions [7]. Some illness perceptions, namely beliefs about personal control and understanding, were significantly associated with glycaemic control $[2,8]$.

People who hold stronger beliefs that their diabetes is chronic and with serious consequences reported a higher emotional impact of their disease; lower perceived personal control was associated with increased depression and anxiety in both type 1 and 2 diabetes patients [6]. Perceived control over diabetes partially mediated the relationship between diabetes-related distress and poorer treatment adherence and glycaemic control among adults with T2DM [9]. The perceived impact of diabetes 4 months after the diagnosis remained a significant predictor of distress and depression at 3-year follow-up [10]. Patients' perception of their illnesses and related symptoms and their beliefs about the possible consequences of the disease are also associated with their satisfaction with medical consultation and healthcare utilisation, respectively [11].

Malaysian data showed an increase in the prevalence of T2DM, a high number of diabetes-related complications and persistent poor disease control and management among T2DM patients [12, 13], especially among the Malays [14]. Given the importance of illness perceptions for health behaviour and outcomes and given the rising burden of T2DM and its associated mood disorders in Malaysia [15, 16], we translated and validated a Malay version of the Brief Illness Perception Questionnaire (MBIPQ) to facilitate the assessment of illness perceptions in a Malay-speaking population. In this study three questions are addressed:

1. What is the cross-cultural validity and construct validity of the MBIPQ in Malay-speaking patients with T2DM?

2. What is the discriminant validity (hypotheses testing) of the MBIPQ in relation to glycaemic control, diabetes-related distress and diabetesrelated complications?

3. What is the intrarater test-retest reliability of the MBIPQ in clinical practice?

The first two questions are about the construct validity and the third question is about reliability of MBIPQ [17]. These three questions are chosen because they are important and fundamental when deciding on using the MBIPQ in practice and before further testing on its other psychometric properties such as responsiveness. Since the BIPQ is considered to be a multiple singleitem measures assessing different aspects of a complex phenomenon, namely perception of diabetes (see below), its factor structure, item-total correlation or internal consistency are considered to be less relevant and usually not tested [18]. In most studies, the original English version of BIPQ has only been on test-retest reliability, discriminant validity, predictive validity and concurrent validity with the more extensive Illness Perception Questionnaire-Revised (IPQ-R) [19], and not on the factor structure or its dimensionality using the item response theory or Rasch approaches [18]. Therefore, we also decided not to explore the internal consistency or factor structure of the MBIPQ. Since content and face validity of BIPQ was already established, we also decided not to describe content and face validity in this study as we translated an existing and fully developed BIPQ. Instead, we focus on cross-cultural validity, construct validity, discriminant validity and intrarater test-retest reliability of each item [17].

\section{Methods}

The 9-item brief illness perception questionnaire (BIPQ)

The BIPQ was designed to provide simple and rapid assessment of illness perceptions. It was developed for use in clinical practice and consists of nine items. These nine items are as below, and available at http:// www.uib.no/ipq/pdf/B-IPQ-English.pdf (Additional file 1). Like the IPQ-R, the general version of the BIPQ uses the word 'illness', but it is possible to replace this with the name of a particular illness such as T2DM or diabetes mellitus and this was done for the MBIPQ.

1. (Consequences) How much does your diabetes affect your life? 
2. (Timeline) How long do you think your diabetes will continue?

3. (Personal Control) How much control do you feel you have over your diabetes?

4. (Treatment Control) How much do you think your treatment can help your diabetes?

5. (Identity) How much do you experience symptoms from your diabetes?

6. (Concern) How concerned are you about your diabetes?

7. (Understanding) How well do you feel you understand your diabetes?

8. (Emotional Response) How much does your diabetes affect you emotionally? (e.g. does it make you angry, scared, upset or depressed)?

9. (Causal Representation) Please list in rank-order the three most important factors that you believe caused your diabetes. The most important causes for me:1)_2)_3)_

All items were developed by forming one question that best summarised the items contained in each subscale of the IPQ-R [20]. Five items assess cognitive illness representations: perceived consequences (Item 1), timeline (acute-chronic) (Item 2), personal control (Item 3), treatment control (Item 4), and the presence of symptoms or identity (Item 5). Two items assess emotional representations: concern (Item 6) and emotions (Item 8) about the illness. One item assesses illness comprehensibility or coherence of the illness (Item 7). In contrast to the more traditional method of constructing dimensions by forming subscales, the BIPQ has just one single item to assess each dimension, rated on a 0-to-10 scale like in the IPQ-R. Higher scores indicate stronger perceptions along that dimension. The BIPQ has been shown to have good psychometric properties in 36 countries and many illness populations [2]. The test-retest reliability correlation coefficients of the original English BIPQ were between 0.48 to 0.70 at 3-weeks and 0.42 to 0.75 at 6 weeks [19]. The equivalent scales of the BIPQ and the IPQ-R are moderately correlated (0.32 to 0.62$)$, including the causal representation item with $75 \%$ of all causes categorised within the 20 causal factors [19]. The personal control item was significantly correlated with diabetes self-efficacy $(r=0.61, p<0.001)$ and lower HbA1c $(r=-0.30, p<0.01)$ [19]. In a systematic review in 2015 [2], pooled correlations were undertaken based on the Fisher's z transformation of the correlation coefficients, showed that between illness perceptions and depression, blood glucose levels and quality of life were in the range 0.25 to 0.49 for consequences, identity and emotional representations and between -0.15 to -0.27 for the personal control item. All items were able to predict some outcomes up to one-year follow-up [2]. Personal control and causal items showed most frequent changes after intervention in randomised controlled trials [2].

Besides these single items, the BIPQ has a part of the causal scale previously used in the IPQ-R [19]. Assessment of the causal representation is by an open-ended response item, which asks patients to list the three most important causal factors of their illness (Item 9). Because each item of the BIPQ assesses one dimension of illness perceptions, the consequences score is simply the response to item 1 et cetera. Reponses to item 9 can be grouped into categories such as lifestyle (food and physical exercise), hereditary, stress, etc.

\section{Setting}

This study was conducted in 2016 in 11 public health clinics in Malaysia, three in Selangor and eight in Negeri Sembilan. These are governmental health clinics in urban, suburban and rural areas that have resident doctors and are headed by family medicine specialists. They provide primary medical care collaborating with a multidisciplinary team of a nutritionist or dietician, pharmacist, physiotherapist, occupational therapist and paramedics who have undergone specialised training in diabetes education and eye care [21].

\section{Study samples}

We used the data of 312 patients with T2DM for this study. They came from two different samples. This number is considered sufficient as the required sample size for a validation study is the number of items $\times 10$ $[18,22]$. Patients were included in the study according to the following criteria: patients who understand Malay, at least 30 years old, diagnosed with T2DM for at least 3 years and with a regular follow-up with three or more visits in the past year. Patients who were pregnant or breastfeeding, or those with severe health problems or psychiatric/psychological disorders that caused cognitive impairments or those who cannot self-administer or be interviewed to complete the questionnaires were excluded. Severe health problems such as life-threatening diseases, recent acute complications or injuries and a recent discharge from hospital comprised the other exclusion criteria. The definition of T2DM was based on: (i) a documented diagnosis of diabetes mellitus according to the World Health Organization criteria or (ii) current treatment consisting of lifestyle modification, oral antihyperglycaemic agents or insulin. Approval was obtained from the original author to use the BIPQ English version. All subjects had provided written informed consent before participation.

\section{Instrument translation and cross-cultural validation}

The translation and adaptation process of the BIPQ from its original language (English) to Malay is shown in 
Fig. 1. It was conducted according to guidelines for cross-cultural adaption of self-report measures [23, 24]. The cultural adaptation process included: (1) review of the original and the two translated questionnaires by an expert committee that was composed of researchers, three family medicine specialists, a psychologist, a methodologist, and three adults with T2DM; except one researcher and the methodologist, the main researcher, the three family medicine specialists, the psychologist, and three adults with T2DM were bilingual (Malay and English) and the three family medicine specialists and the three adults with T2DM were native language (Malay) speakers; (2) reconciliation, (3) harmonisation, (4) cognitive debriefing, (5) review of cognitive debriefing results and finalisation, (6) proofreading and (7) final report.

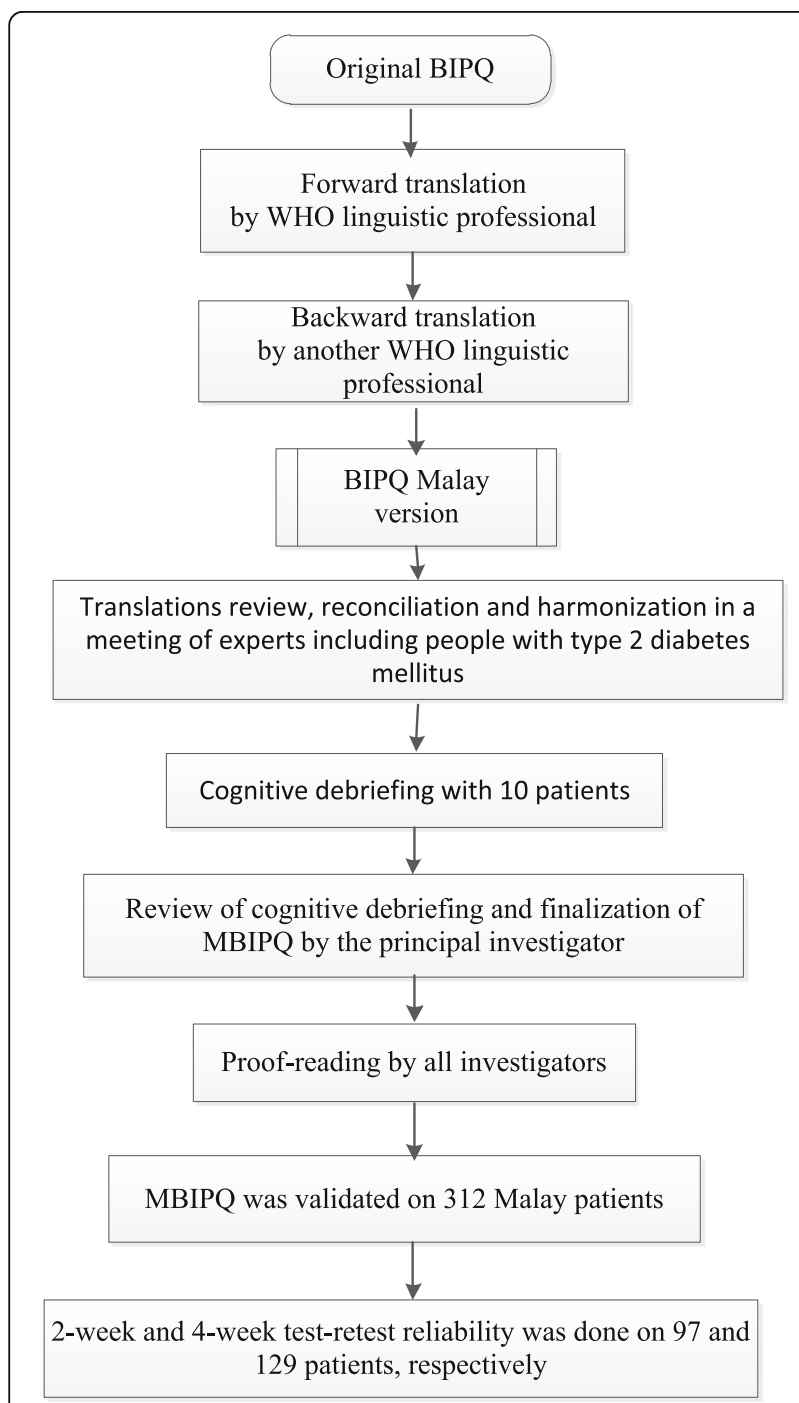

Fig. 1 Translation and validation process. BIPQ = Brief IIIness Perception Questionnaire. MBIPQ = Malay version of the Brief Illness Perception Questionnaire. WHO = World Health Organization
This version of the MBIPQ was distributed to 10 Malay patients with the above mentioned inclusion and exclusion criteria for comment. These individuals were not included in the later validation study. They commented that the items were easily understood and acceptable with regards to their cultural background. Therefore, that MBIPQ version was accepted without modification for the validation study.

\section{Assessing construct validity}

Construct validity for the eight items in MBIPQ, as a measure of different illness perceptions, was assessed by comparing self-efficacy, quality of life, medication adherence, diabetes-related distress and depressive symptoms with each item of the MBIPQ. For that purpose, patients self-administered the socio-demographic and MBIPQ questionnaires, and for those who could not, face-toface interviews were performed by trained research assistants. Additionally, they completed a set of the below mentioned five questionnaires:

1. The 20-item Diabetes Management Self Efficacy Scale (DMSES) measures patients' confidence in managing their disease in terms of blood glucose, diet, and exercise, with items on an 11-point Likert scale, scores ranging from 0 if they "cannot do at all" to 10 if the respondents "certainly can do"; lower scores indicating low self-efficacy for coping with the activities listed [25-27]. In social cognitive theory of self-regulation $[28,29]$, self-efficacy plays important roles in health behaviours through its influences on personal thoughts, emotion, motivation and action. Previous research has shown significant moderate correlations between diabetes self-efficacy and perceived control $(r=0.61)$ [19], and we expected to find similar correlations between the MBIPQ personal control item and self-efficacy.

2. The World Health Organization Quality of Life-brief version (WHOQOL-BREF), 25 items measuring quality of life over the past 4 weeks in the physical, psychological, social relationships and environmental domain, with scores from 25 to 100 and higher scores denoting higher quality of life) [30-32]. WHOQOL-BREF assesses activities of daily living, thinking, learning, environment safety, freedom to move about, feelings and dependence on medical therapies which are influenced by health beliefs and personal illness perception as measured by MBIPQ. Previous studies showed that higher consequences $(r=0.37$ to 0.49$)$, emotional representation and identity ( $r=0.34$ to 0.43 ) were associated with lower quality of life; and personal control $(r=0.18$ to 0.22$)$ and treatment control $(r=0.16)$ had significant correlations with better quality of life [2]. We expected 
to find similar correlations in MBIPQ with this quality of life measure.

3. The 8-item Morisky Medication Adherence Scale (MMAS-8), measuring medication adherence during the past 2 weeks, and with scores from 0 to 8 , resulting in the following levels of adherence: low $(<6)$, medium (6,7) and high (8) [33-36]. MMAS-8 allows a construct assessment of the treatment control item in MBIPQ [37, 38]. Based on literature, we expected to find a positive correlation (approximately $r=0.20$ ) between a higher belief in treatment as well as a higher perception of personal control on the one hand and medication adherence on the other $[39,40]$.

4. The 17-item Diabetes Distress Scale (DDS-17) assesses problems and hassles concerning diabetes during the last month. Each item is scored on a Likert scale from 1 (not a problem) to 6 (a very serious problem). It yields a total scale score with a mean total score of $\geq 3$ (severe distress) is considered a level of distress worthy of clinical attention [41, 42]. Previous studies showed that distress influences perceptions of self-management and were associated with poor diabetes-treatment adherence and HbA1c [9], lower self-efficacy, and poorer dietary and exercise behaviours [43-45]. Examining the correlations between the emotional items of the MBIPQ with diabetes-related distress as measured with the DDS-17 in order to test the construct validity of the MIBQ, we expected to find correlations of approximately $r=0.40$ between both the items 'consequences', 'concern' and 'emotional response' on the one hand and diabetes-related distress [46].

5. The 9-item Patient Health Questionnaire (PHQ-9) $[47,48]$ evaluates depressive symptoms and grades the depression severity. It scores from 0 to 27, which can be classified as 0-4 (minimal), 5-9 (mild), 10-14 (moderate), 15-19 (moderately severe), and 20-27 (severe) $[47,48]$. Previous studies with the BIPQ showed that higher consequences $(r=0.41)$, emotional response $(r=0.39)$ and identity $(r=0.32)$ were associated with higher depression; personal control $(r=-0.15)$ and treatment control $(r=-0.11)$ had small negative correlations with depression [2]. We expected to find similar correlations between MBIPQ with depressive symptoms.

\section{Discriminant validity}

The discriminant validity of the MBIPQ was examined in relation to HbA1c, an estimate of blood glucose control over the past 3 months; and also by its ability to distinguish between patients with severe and non-severe diabetesrelated distress (mean DDS-17 score $\geq 3$ versus $<3$ ) [43] and between those with and without diabetes-related complications. HbA1c results within the past 3 months were retrieved from medical records. This is analysed in the respective health clinics or regional hospitals with government approved analysers that are calibrated on annual basis. Diabetes-related complications were also retrieved from the medical records, the diagnoses of these conditions are based on clinical practice guidelines.

\section{Intrarater test-retest reliability}

To examine the test-retest reliability, patients in the eight public health clinics in Negeri Sembilan were invited for a 2-week test-retest and those in the three Selangor public health clinics for a 4-week re-test, respectively. The former were participants in a trial that had an interval of 2 weeks between their baseline meeting and first session of the programme, the latter would return to the clinic for medication re-supply. Data collection procedures were similar and standardised between test and retest. Participants in Negeri Sembilan answered the MBIPQ before the first session of the programme, whereas those in Selangor completed MBIPQ in the waiting area of the health clinics while waiting for a doctor's consultation.

\section{Statistical analyses}

Descriptive statistics were used to describe the demographic and disease characteristics of the patients and their MBIPQ item scores. The mean (SD) scores of the first eight items of the MBIPQ were reported at the first, the 2-week and 4-week testing. Floor and ceiling effects at each testing moment were also reported. To assess the construct validity of the MBIPQ the following analyses were performed: Spearman's or Pearson's correlation coefficients were calculated and Student t-tests were carried out for MBIPQ item scores with self-efficacy, quality of life, medication adherence, diabetes-related distress and depressive symptoms as categorical and continuous independent variables, respectively. The discriminant validity of the MBIPQ was first examined by measuring the Pearson's or Spearman's between each item of the MBIPQ and $\mathrm{HbA1c}$. To distinguish between patients with severe and non-severe diabetes-related distress and between those with and without diabetes-related complications, Student t-tests or the Mann-Whitney U test were performed.

Pearson's correlations were calculated for normally distributed and for non-normally distributed variables (items/scales) if Spearman's correlations gave similar results. Otherwise, Spearman's correlations were reported. Comparing the associations of severe / non-severe diabetes-related distress, and of diabetes-related complication / no complication with all the MBIPQ item scores, the Student $\mathrm{t}$-tests and the Mann-Whitney $\mathrm{U}$ test showed similar results (except item 3). We report the association with Student t-test. Correlations were interpreted using 
the following Cohen's criteria: $0-0.25=$ little or no correlation, $0.25-0.5=$ fair correlation, $0.5-0.70=$ moderate to good correlation and $>0.70=$ very good to excellent correlation [49].

Since the MBIPQ uses a single-item scale approach to assess perceptions on a continuous linear scale, we used intraclass correlation coefficients ( $\mathrm{ICC}_{\text {agreement }}$ ) of twoway random effects model to assess test-retest reliability [18] at 2-week and 4-week. This will take into account systematic differences that Pearson's or Spearman's correlation does not [18]. ICC $\mathrm{I}_{\text {agreement }}$ of 0.70 is recommended as a minimum standard for reliability [18]. Besides, the three most common causes for T2DM in item 9 of the MBIPQ were presented. As these causes were ordinal and ranked, we used Spearman's correlation to examine the correlation of the causes that were ranked as first, second and third between the first and the repeated responses at 2-week and 4-week. Statistical analyses were performed using Statistical Package for Social Science 22.0 (SPSS, Chicago, IL). The significance level was set at $p<0.05$.

\section{Results}

A total of 324 patients (100 of 123 from Negeri Sembilan and 224 of 361 from Selangor) with T2DM responded but 312 patients (97 of 100 from Negeri Sembilan and 215 of 224 from Selangor) completed the MBIPQ. The age of the non-responders was significantly older than the participants $(61.7$ vs. 58.2 years, respectively, $\mathrm{t}$ test $=3.28$, $p=0.001$ ) and gender (male sex 44.4 and $42.3 \%$, respectively, $X^{2}=0.18, p=0.680$ ) was not significantly different between the two groups. All the 97 patients from Negeri Sembilan completed the 2-week test-retest and 129 patients from Selangor completed the test-retest after 4 weeks. The demographic characteristics of the participants are presented in Table 1.

\section{Construct validity}

Table 2 shows the mean (SD) of the eight items in MBIPQ at each testing. Floor effects, with more than $15 \%$ of the participants mentioning the lowest possible score [18] were seen in the item understanding (19.4\%) at the first assessment. The ceiling effects, with more than $15 \%$ of the participants mentioning the highest possible score [18] were seen in the items timeline (35.3\%) and concern $(26.1 \%)$ at the first assessment, and again in item timeline $(27.7 \%)$ at the 4 -week retest.

Table 3 shows that illness perceptions correlated in a logical way, at best moderate: perceiving symptoms (identity) was significantly related to being affected emotionally by the diabetes (emotional response) $(r=0.53)$ and being concerned about their T2DM was related to perceiving consequences of the disease $(r=0.42)$. Significant correlations were also noted between perceived personal $(r=0.31)$ as well as treatment control $(r=0.35)$ and understanding of the illness. Conversely, those who understood more about their T2DM also expressed lesser concern $(r=-0.30)$. Negative correlations between timeline and perceived personal control $(r=-0.23)$, and timeline and perceived treatment control $(r=-0.21)$, indicated that perception of chronicity was associated with loss of perceived personal control and treatment ineffectiveness, respectively.

Overall, there were 71 and 141 participants who did not provide the cause for their T2DM in the first and repeated MBIPQ, respectively. The three most common causes for T2DM listed by the participants in the first MBIPQ were dietary (283/603), sedentary lifestyle (114/603) and hereditary $(108 / 603)$. Other causes $(98 / 603)$ mentioned at least 10 times included obesity (16), emotional conditions such as stress (16), lack of health-related knowledge (16), medication (10) and lifestyle factor (10). There were significant correlations of the first $(r=0.35, p=0.003, n=70)$, second $(r=0.30, p=0.031, n=53)$ and third $(r=0.37, p=0.021$, $n=38$ ) important causes as mentioned in the 2-week interval between the first and second measurement. However, there were no significant correlations noted for all three important causes in the 4-week interval.

Table 4 shows a number of correlations between MBIPQ items and patient-reported outcomes. Some correlations were in the expected range of approximately 0.20 , namely three out of eight with self-efficacy, two with medication adherence, five with quality of life, five with depressive symptoms and seven with diabetesrelated distress. This indicates a fair construct validity (hypotheses testing) of the MBIPQ. Those who understood their T2DM well or had a higher perception of personal or treatment control had a higher self-efficacy. Similarly, patients who had a higher personal control or perceived treatment effectiveness according to the MBIPQ were more adherent to their medication, experiencing better quality of life, a lower HbAlc and less depressive symptoms. Significant correlations were also noted between item Identity (experiencing diabetic symptoms) and less self-efficacy, lower quality of life and more depressive symptoms.

\section{Discriminant validity}

Patients who had a higher personal control or perceived treatment effectiveness according to the MBIPQ had a lower HbA1c (Table 4). The relationship between the MBIPQ items and the total diabetes-related distress score is also depicted in Table 4. Those who had higher diabetesrelated distress were generally having a threatening view about T2DM (except understanding). Those who had severe diabetes-related distress (DDS-17 scores $\geq 3$ ) were perceiving more consequences (t-test $=3.20, p=0.002$ ), identity (t-test $=2.97, p=0.003)$ and concern (t-test $=4.01$, 
Table 1 Sample characteristics

\begin{tabular}{|c|c|c|c|}
\hline & Validation, $n=312$ & $\begin{array}{l}\text { Negeri Sembilan 2-week } \\
\text { test-retest reliability, } n=97\end{array}$ & $\begin{array}{l}\text { Selangor } 4 \text {-week test-retest } \\
\text { reliability, } n=129\end{array}$ \\
\hline Age, mean $(S D)^{*}$ & $58.1(9.67)$ & $56.1(9.10)$ & $59.1(9.61)$ \\
\hline \multirow[t]{2}{*}{ Diabetes duration in years, mean (SD) } & $8.5(4.79)$ & $8.5(5.92)$ & $8.3(4.15)$ \\
\hline & n (\%) & & \\
\hline \multicolumn{4}{|l|}{ Gender } \\
\hline Female & $184(59.0)$ & $61(62.9)$ & $69(53.5)$ \\
\hline Male & $128(41.0)$ & $36(37.1)$ & $60(46.5)$ \\
\hline \multicolumn{4}{|l|}{ Ethnicity $^{a}$} \\
\hline Malay & $240(76.9)$ & $97(100)$ & $87(67.4)$ \\
\hline Chinese & $9(2.9)$ & 0 & $9(7.0)$ \\
\hline Indian & $61(19.6)$ & 0 & $32(24.8)$ \\
\hline \multicolumn{4}{|l|}{ Educational level $^{a}$} \\
\hline No school & $15(4.9)$ & 0 & $9(7.1)$ \\
\hline Primary school level & $99(32.2)$ & $14(14.6)$ & $55(43.3)$ \\
\hline Secondary school level & $161(52.4)$ & $70(72.9)$ & $56(44.1)$ \\
\hline Tertiary school level & $32(10.4)$ & $12(12.5)$ & $7(5.5)$ \\
\hline \multicolumn{4}{|l|}{ Life event in the past 6 months $^{a}$} \\
\hline Yes & $61(19.7)$ & $27(28.4)$ & $16(12.4)$ \\
\hline No & $248(80.3)$ & $68(71.6)$ & $113(87.6)$ \\
\hline \multicolumn{4}{|l|}{ Co-morbidity } \\
\hline Hypertension & $269(86.2)$ & $84(86.6)$ & $108(83.7)$ \\
\hline Dyslipidaemia & $256(82.1)$ & $84(86.6)$ & $107(82.9)$ \\
\hline Any diabetes complication ${ }^{a}$ & $98(31.4)$ & $25(25.8)$ & $51(39.5)$ \\
\hline \multicolumn{4}{|l|}{ Treatment } \\
\hline Oral hypoglycaemic agent ${ }^{a}$ & $266(85.3)$ & $94(96.9)$ & $103(79.8)$ \\
\hline Insulin ${ }^{a}$ & $161(51.6)$ & $74(76.3)$ & $58(45.0)$ \\
\hline $\mathrm{HbA} 1 \mathrm{c}$ in $\%$, mean $(\mathrm{SD})^{\mathrm{a}}$ & $9.0(2.27)$ & $9.7(1.99)$ & $8.9(2.43)$ \\
\hline
\end{tabular}

$S D$ standard deviation

${ }^{\text {a }}$ Statistically significant differences between the Negeri Sembilan and Selangor at $p$ value $<0.05$

Table 2 Mean (SD) scores on eight items of the Malay Brief Illness Perception Questionnaire at each testing

\begin{tabular}{llll}
\hline MBIPQ Item (Min. - Max.) & 0 week & 2 weeks & 4 weeks \\
\hline Consequences (0-10) & $4.7(2.63)$ & $4.4(2.54)$ & $5.0(2.14)$ \\
Timeline (0-10) & $7.2(2.86)$ & $6.2(2.42)$ & $8.2(2.29)$ \\
Personal Control (0-10) & $2.9(2.07)$ & $3.1(2.13)$ & $3.0(1.73)$ \\
Treatment Control (0-10) & $1.8(1.88)$ & $2.4(2.09)$ & $1.6(1.79)$ \\
Identity (0-10) & $4.8(2.61)$ & $5.0(2.28)$ & $5.3(2.08)$ \\
Concern (0-10) & $6.9(2.87)$ & $6.4(2.67)$ & $6.7(2.43)$ \\
Understanding (0-10) & $2.6(2.22)$ & $2.7(2.08)$ & $2.9(1.85)$ \\
Emotional Response (0-10) & $5.2(2.82)$ & $5.2(2.57)$ & $5.3(2.49)$ \\
\hline
\end{tabular}

$p<0.001$ ), and lower personal control (t-test $=2.07$, $p=0.031)$. Perceiving the diabetes treatment not effective (t-test $=2.39, p=0.017$ ) was also associated with more severe diabetes-related distress. Patients with and any diabetes-related complication were also having higher consequences (t-test $=2.04, p=0.044)$ and identity scores (t-test $=2.86, p=0.005)$.

\section{Reliability}

Table 5 shows the intrarater test-retest correlations after 2- and 4-week. The 2-week test-retest reliability

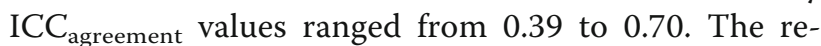
spective values after 4 -weeks are 0.58 to 0.78 , with the highest for concern.

\section{Discussion}

MBIPQ provides a rapid and moderately good assessment of the personal beliefs that patients hold about 
Table 3 Items inter-correlations (Pearson's correlation coefficients) of the Malay Brief Illness Perception Questionnaire in adults with type 2 diabetes mellitus

\begin{tabular}{|c|c|c|c|c|c|c|c|c|}
\hline & \multicolumn{8}{|c|}{ Pearson's correlation, $r(n)$} \\
\hline & Consequences & Timeline & $\begin{array}{l}\text { Personal } \\
\text { Control }\end{array}$ & $\begin{array}{l}\text { Treatment } \\
\text { Control }\end{array}$ & Identity & Concern & Understanding & $\begin{array}{l}\text { Emotiona } \\
\text { Response }\end{array}$ \\
\hline Consequences & $1(324)$ & & & & & & & \\
\hline Timeline & $0.233^{* *}(320)$ & $1(320)$ & & & & & & \\
\hline Personal Control & $0.090(322)$ & $-0.231^{* *}(318)$ & $1(322)$ & & & & & \\
\hline Treatment Control & $0.082(322)$ & $-0.211^{* *}(319)$ & $0.505^{* *}(320)$ & $1(322)$ & & & & \\
\hline Identity & $0.503^{* *}(321)$ & $0.259^{* *}(318)$ & $0.072(319)$ & $0.016(320)$ & $1(321)$ & & & \\
\hline Concern & $0.418^{* *}(322)$ & $0.131^{*}(318)$ & $-0.042(320)$ & $-0.058(320)$ & $0.379^{* *}(319)$ & $1(322)$ & & \\
\hline Understanding & $-0.087(324)$ & $-0.142^{*}(320)$ & $0.314^{* *}(322)$ & $0.352^{* * *}(322)$ & $-0.060(321)$ & $-0.303^{* *}(322)$ & $1(324)$ & \\
\hline Emotional Response & $0.525^{* *}(322)$ & $0.138^{*}(318)$ & $0.009(320)$ & $0.001(320)$ & $0.532^{* *}(320)$ & $0.514^{* *}(320)$ & $-0.165^{* *}(322)$ & $1(322)$ \\
\hline
\end{tabular}

their T2DM. Thus, MBIPQ may be of value for clinical practice as a starting point in clinical consultations to talk about patient's worries about T2DM, feeling overwhelmed and beliefs in treatment options. This would allow a more tailored treatment and support from the healthcare team. This study provided a translated and culturally adapted Malay version of the BIPQ. Interitems correlations were at best moderate, and correlations with the studied patient-reported outcomes were at best fair according to the Cohen's criteria. The discriminant validity of the MBIPQ was supported by its ability to distinguish between patients with different levels of diabetes-related distress and diabetes-related complications. The intrarater test-retest reliability was good for a few items but less than the recommended standard [18] between most items.

MBIPQ has moderate construct validity as some items showed the expected hypothesised correlation coefficients with the patient-reported outcome measures. Correlations between the MBIPQ treatment and personal control items and self-efficacy, medication adherence, quality of life and emotional distresses are in line with those of former studies $[2,39,40]$. The correlation coefficient between perceived personal control and diabetes self-efficacy was about half of that observed in the original English BIPQ study which was measured with the Multidimensional Diabetes Questionnaire $(r=0.61$, $p<0.001$ ) [19]. Other cognitive perceptions also showed the expected association with medication adherence indicating that those who perceived and experienced T2DM in a more positive manner also tend to adhere to their medication. We could not demonstrate significant associations between timeline with quality of life and depression, in contrast to a meta-analysis [2] that showed that a higher perception of chronicity of diabetes was associated with on the one hand better quality of life, but on the other a higher depression rate, more anxiety and higher HbA1c. However, the association between timeline and diabetes-related distress was negative, indicating that those who believe that T2DM is of short duration are experiencing higher diabetes-related distress. It is difficult to explain this association in this study although

Table 4 Correlation (Pearson's correlation coefficients) between MBIPQ and self-efficacy, medication adherence, quality of life, depressive symptoms, diabetes-related distress and $\mathrm{HbA} 1 \mathrm{C}$

\begin{tabular}{|c|c|c|c|c|c|c|c|}
\hline No. & MBIPQ Item & Self-efficacy & Medication adherence & Quality of life & Depressive symptoms & Total DDS-17 & $\mathrm{HbA} 1 \mathrm{c}$ \\
\hline 1. & Consequences & -0.05 & -0.09 & $-0.17^{* *}$ & $0.21^{* *}$ & $0.28^{* *}$ & 0.10 \\
\hline 2. & Timeline & 0.10 & $0.10^{\mathrm{a}}$ & 0.05 & $-0.11^{b}$ & $-0.15^{* *}$ & -0.09 \\
\hline 3. & Personal Control & $0.35^{* *}$ & $0.23^{* *}$ & $0.31^{* *}$ & $-0.12^{*}$ & $-0.23^{* *}$ & $-0.13^{*}$ \\
\hline 4. & Treatment Control & $0.26^{* *}$ & $0.29^{* *}$ & $0.31^{* *}$ & $-0.18^{* *}$ & $-0.17^{* *}$ & $-0.16^{* *}$ \\
\hline 5. & Identity & $-0.13^{*}$ & -0.04 & $-0.17^{*}$ & $0.16^{* *}$ & $0.23^{* *}$ & 0.09 \\
\hline 6. & Concern & 0.05 & -0.04 & -0.09 & $0.21^{* *}$ & $0.28^{* *}$ & $0.23^{* *}$ \\
\hline 7. & Understanding & $0.35^{* *}$ & -0.06 & $0.14^{*}$ & -0.03 & 0.004 & $0.12^{*}$ \\
\hline 8. & Emotional Response & -0.02 & -0.10 & $-0.18^{* *}$ & $0.29^{* *}$ & $0.28^{* *}$ & $0.15^{* *}$ \\
\hline
\end{tabular}

MBIPQ Malay version of the Brief Illness Perception Questionnaire, DDS-17 the 17-item Diabetes Distress Scale

${ }^{*} p$ value $<0.05,{ }^{* *} p$ value $<0.01$

a Spearman correlations was 0.15 at $p=0.010$

${ }^{\mathrm{b}}$ Spearman correlations was -0.20 at $p<0.01$ 
Table 5 Intraclass correlation coefficients of the Malay Brief Illness Perception Questionnaire

\begin{tabular}{lll}
\hline MBIPQ Item, $(n)$ & 2 weeks, $(n)$ & 4 weeks, $(n)$ \\
\hline Consequences $(n=324)$ & $0.677^{* *}(n=100)$ & $0.680^{* *}(n=133)$ \\
Timeline $(n=320)$ & $0.697^{* *}(n=98)$ & $0.582^{* *}(n=133)$ \\
Personal Control $(n=322)$ & $0.387^{*}(n=99)$ & $0.685^{* *}(n=133)$ \\
Treatment Control $(n=322)$ & $0.569^{* *}(n=100)$ & $0.671^{* *}(n=133)$ \\
Identity $(n=321)$ & $0.498^{* *}(n=100)$ & $0.664^{* *}(n=132)$ \\
Concern $(n=322)$ & $0.687^{* *}(n=100)$ & $0.781^{* *}(n=131)$ \\
Understanding $(n=324)$ & $0.462^{* *}(n=100)$ & $0.664^{* *}(n=132)$ \\
Emotional Response $(n=322)$ & $0.652^{* *}(n=100)$ & $0.610^{* *}(n=132)$ \\
\hline
\end{tabular}

${ }^{*} p$ value $<0.05,{ }^{* *} p$ value $<0.01$

age could be the confounding factor $[15,50]$. It seems plausible that those who experienced much symptoms (identity) expressed lower self-efficacy, lower quality of life and more depressive symptoms. The same applies to MBIPQ items that showed expected results with the diabetes-related distress. Patients who perceived better personal or treatment control demonstrated less depressive symptoms and less diabetes-related distress while those who were distressed in this respect showed high concern and emotional response to T2DM. The associations between perceptions of better personal or treatment control and less depressive symptoms and a lower HbA1c are in concordance with earlier studies [2, 51]. Our finding that more concern and emotional perceptions were significantly associated with higher HbAlc are in line with the results of previous studies [51]. However, in contrary to past studies MBIPQ did not show consequences and identity beliefs to have any significant correlation with HbA1c [2].

Intraclass correlation coefficients were almost all below 0.70. In our opinion this does not mean that the MBIPQ is an unreliable measure. Illness perceptions as assessed by the MBIPQ are susceptible to change, for example after an intervention [2]. Sensitivity to change of the MBIPQ needs to be studied further. The similar or even slightly better 4-week test-retest reliability compared to the 2-week reliability test might be due to the two different cohorts of respondents in this study. The people from Negeri Sembilan experienced more life events in the past 6 months (Table 1) and had more emotional distresses [52]; these differences might have led to fluctuation in illness perceptions through impairment of self-management ability [53] and cognitive function [54]. Obviously, this will result in lower intrarater test-retest correlations, as we found. Because we used $\mathrm{ICC}_{\text {agreement }}$ for the test-retest, it is hard to compare the test-retest reliability with the results of the other BIPQ versions that calculated Pearson's correlation such as the Chinese version ( $r=0.24$ to 0.76$)$ and the Farsi version $(r=0.50$ to 0.75$)$ [8] that were retested after 4 weeks
[55], the original English version that was retested after 3 weeks $(r=0.48$ to 0.70$)$ and 6 weeks $(r=0.42$ to 0.75$)$ [19]. Nevertheless, the test-retest reliability of the MBIPQ is found to be satisfactory and more or less comparable to the other versions [56, 57].

\section{Strength and limitations}

Standard translation with rigorous adaptation procedures by an expert committee that included patients with T2DM had produced the MBIPQ of good crosscultural validities. Further, adequate sample size and variety of the respondents were the strength of this study. Construct and discriminant validation were done with multiple validated and well known outcomes. However, having nine items instead of one index or summary score to handle in statistical models may be a major limitation of (M)BIPQ. We could not handle MBIPQ as a single construct (a reflective model in which all items are a manifestation of the same underlying construct) because of the low inter-items correlations in our study population. We may consider the MBIPQ as a formative measure if we assume that all item scores will change after for example an education on diabetes selfmanagement [58]. In addition, adding up items gets information lost about which perceptions are most strongly linked to the studied outcomes such as between personal control and understanding and self-efficacy, between treatment control and medication adherence, etc. (data not shown). Another limitation is the lack of equivalent scales (such as knowledge on T2DM) for some of the illness perception dimensions which hinders a full investigation of MBIPQ construct validity. Due to the inclusion criteria the results of this study are less applicable to the Chinese community in Malaysia and to T2DM patients who are treated in a secondary care setting. Future research is needed to establish its properties on responsiveness and predictive validity.

\section{Conclusions}

The psychometric properties of items in the MBIPQ are moderate. The MBIPQ showed good content and face validity. Construct validity showed small but significant correlations for all illness perception dimensions with relevant outcome measures, and test-retest reliability was generally moderate. The discriminant validity of the MBIPQ was supported by its ability to distinguish between categories of patients with different categories of diabetes-related distress and diabetes related complications. For that reason we think the MBIPQ may be useful in clinical practice, it offers an opportunity to assess and potentially modify people's perceptions, understanding and experience of T2DM. However, the timeline and concern items do not perform well and should be used with most caution. 


\section{Additional file}

Additional file 1: Two questionnaires. The first one is the Malay version of the Brief IIIness Perception Questionnaire (BIPQ), and the second one is the English translation of the BIPQ. (PDF $89 \mathrm{~kb}$ )

\begin{abstract}
Abbreviations
BIPQ: The 9-item Brief IIIness Perception Questionnaire; DDS-17: 17-item Diabetes Distress Scale; DMSES: Diabetes Management Self Efficacy Scale; IPQ-R: Illness Perception Questionnaire-Revised; MBIPQ: Malay version of the Brief Illness Perception Questionnaire; MMAS-8: 8-item Morisky Medication Adherence; PHQ-9: 9-item Patient Health Questionnaire; T2DM: Type 2 diabetes mellitus; WHOQOL-BREF: World Health Organization Quality of Life-brief
\end{abstract}

\section{Acknowledgements}

We acknowledge the Director General of Health Malaysia for their permission to publish this report. We thank Universiti Putra Malaysia and the Ministry of Higher Education Malaysia for sponsoring the PhD study of BHC. The Seremban, Petaling and Sepang District Health Offices are acknowledged for their support. The following family medicine specialists are acknowledged: Ziti-Akthar Supian, Noor-Hasliza Hassan and Husni Hussain, Mastura Ismail, Azainorsuzila Mohd Ahad, Iliza Idris, Narayanan N. Sundram, Siti Zubaidah Mohd Ali, Nor Asiah Hashim, Sabariah Idris, Siti Rokiah, Mariam Abdul Manap and Nabiha Samsudin. Use of the OMMAS is protected by US and International copyright laws. Permission for use is required. A license agreement is available from: Donald E. Morisky, MMAS Research (MORISKY) 16636 159th Place SE, Renton WA 98058, dmorisky@gmail.com.

\section{Funding}

This study was partly funded by the Malaysian MOH-NIH Research Grant (MRG) and award number is 00382. The funding bodies have no role in the design of the study and collection, analysis, and interpretation of data and in writing the manuscript.

\section{Availability of data and materials}

The datasets used and/or analysed during the current study available from the corresponding author on reasonable request. The permission to use BIPQ Malay version may be requested from the corresponding author.

\section{Authors' contributions}

BHC drafted the manuscript. BHC, RCV and GEHMR conceived of the design for the study. BHC, RCV, SSG and AF participated in its data collection and coordination. BHC, RCV, MH and GEHMR contributed to analysis and interpretation of data. All authors involved in interpretation of data. All authors helped to read, revise and approved the final manuscript. All authors agreed to be accountable for all aspects of the work. Authorship eligibility is in accordance to the International Committee of Medical Journal Editors (ICMJE) guidelines.

\section{Ethics approval and consent to participate}

This study was approved by the Medical Research Ethics Committee (MREC), Ministry of Health Malaysia with the reference numbers of (5)KKM/NIHSEC/ P16-293 and (10)KKM/NIHSEC/P15-1159. Patients who agreed to participate provided a written informed consent.

\section{Consent for publication}

Not applicable

\section{Competing interests}

The authors declare that they have no competing interests.

\section{Publisher's Note}

Springer Nature remains neutral with regard to jurisdictional claims in published maps and institutional affiliations.

\section{Author details}

${ }^{1}$ Department of Family Medicine, Faculty of Medicine and Health Sciences, Universiti Putra Malaysia, 43400 Serdang, Selangor, Malaysia. ${ }^{2} J u l i u s$ Center for Health Sciences and Primary Care, Department of General Practice,
University Medical Center Utrecht, Huispost Str.6.131, P.O. Box 85500, 3508, GA, Utrecht, The Netherlands. ${ }^{3}$ NIVEL, Netherlands Institute for Health Services Research, Utrecht, Netherlands. ${ }^{4}$ Department of Psychiatry, Faculty of Medicine and Health Sciences, Universiti Putra Malaysia, 43400 Serdang, Selangor, Malaysia.

Received: 19 January 2017 Accepted: 26 July 2017

Published online: 03 August 2017

\section{References}

1. Leventhal H, Benyamini Y, Brownlee S, Michael D, A. L. E, Linda P-M, R. C. Illness representations: theoretical foundations. In: Keith JP, John W, editors. Perceptions of health and illness. Amsterdam: Harwood Academic; 1997. p. 155-88.

2. Broadbent E, Wilkes C, Koschwanez H, Weinman J, Norton S, Petrie KJ. A systematic review and meta-analysis of the brief illness perception questionnaire. Psychol Health. 2015;30(11):1361-85.

3. Harvey JN, Lawson VL. The importance of health belief models in determining self-care behaviour in diabetes. Diabet Med. 2009:26(1):5-13.

4. Weinman J, Petrie KJ. Illness perceptions: a new paradigm for psychosomatics? J Psychosom Res. 1997:42(2):113-6.

5. Parfeni M, Nistor I, Covic A. A systematic review regarding the association of illness perception and survival among end-stage renal disease patients. Nephrol Dial Transplant. 2013;28(10):2407-14.

6. Hudson IL, Bundy C, Coventry PA, Dickens C. Exploring the relationship between cognitive illness representations and poor emotional health and their combined association with diabetes self-care. A systematic review with meta-analysis. J Psychosom Res. 2014;76(4):265-74.

7. Petricek G, Vrcic-Keglevic M, Vuletic G, Cerovecki V, Ozvacic Z, Murgic L. IIness perception and cardiovascular risk factors in patients with type 2 diabetes: cross-sectional questionnaire study. Croat Med J. 2009;50(6):583-93.

8. Bazzazian S, Besharat MA. Reliability and validity of a Farsi version of the brief illness perception questionnaire. Procedia - Social and Behavioral Sciences. 2010:5:962-5.

9. Gonzalez JS, Shreck E, Psaros C, Safren SA. Distress and type 2 diabetestreatment adherence: a mediating role for perceived control. Health Psychol. 2015;34(5):505-13.

10. Skinner TC, Khunti K, Carey ME, Dallosso H, Heller S, Davies MJ. Stability and predictive utility, over 3 years, of the illness beliefs of individuals recently diagnosed with type 2 diabetes mellitus. Diabet Med. 2014:31:1260-3.

11. Donkin L, Ellis CJ, Powell R, Broadbent E, Gamble G, Petrie KJ. Illness perceptions predict reassurance following a negative exercise stress testing result. Psychol Health. 2006;21(4):421-30.

12. Mastura I, Chew BH, Lee PY, Cheong AT, Sazlina SG, Jamaiyah H, Alwi SARS, Sri Wahyu T, Zaiton A. Control and treatment profiles of 70,889 adult type 2 diabetes mellitus patients in Malaysia. International Journal of Collaborative Research on Internal Medicine \& Public Health. 2011;3(1):98-113.

13. Chew BH, Lee PY, Cheong AT, Mastura I, Sazlina SG, Goh PP. Messages from the Malaysian diabetes registries on diabetes Care in Malaysian public healthcare facilities. Prim Care Diabetes. 2016;10(5):383-6.

14. Lee PY, Cheong AT, Zaiton A, Mastura I, Chew BH, Sazlina SG, Adam BM, Syed Alwi SAR, Jamaiyah H, Sri WT. Does ethnicity contribute to the control of cardiovascular risk factors among patients with type 2 diabetes? Asia Pac J Public Health. 2013;25(4):316-25.

15. Chew BH, Vos R, Mohd-Sidik S, Rutten GEHM. Diabetes-related distress, 881 depression and distress-depression among adults with type 2 diabetes 882 mellitus in Malaysia. PLoS One. 2016;11(3):e0152095.

16. Kaur G, Tee GH, Ariaratnam S, Krishnapillai AS, China K. Depression, anxiety and stress symptoms among diabetics in Malaysia: a cross sectional study in an urban primary care setting. BMC Fam Pract. 2013;14:69.

17. Mokkink LB, Terwee CB, Patrick DL, Alonso J, Stratford PW, Knol DL, Bouter LM, de Vet HC. The COSMIN study reached international consensus on taxonomy, terminology, and definitions of measurement properties for health-related patient-reported outcomes. J Clin Epidemiol. 2010;63(7):737-45.

18. Terwee CB, Bot SD, de Boer MR, van der Windt DA, Knol DL, Dekker J, Bouter LM, de Vet HC. Quality criteria were proposed for measurement properties of health status questionnaires. J Clin Epidemiol. 2007;60(1):34-42.

19. Broadbent E, Petrie KJ, Main J, Weinman J. The brief illness perception questionnaire. J Psychosom Res. 2006;60(6):631-7.

20. Moss-Morris R, Weinman J, Petrie KJ, Horne R, Cameron LD, Buick D. The revised illness perception questionnaire (IPQ-R). Psychol Health. 2002;17(1):1-16. 
21. Jaafar S, Mohd Noh K, Abdul Muttalib K, Othman N. H., Healy J. Malaysia health system review. Health Syst Transition. 2013;3(1):15-80.

22. Peat J. Health science research: a handbook of quantitative methods. London: SAGE Publications Ltd; 2002.

23. Wild D, Grove A, Martin M, Eremenco S, McElroy S, Verjee-Lorenz A, Erikson P. ISPOR task force for translation and cultural adaptation. Principles of good practice for the translation and cultural adaptation process for patient-reported outcomes (PRO) measures: report of the ISPOR task force for translation and cultural adaptation. Value Health. 2005;8(2):94-104.

24. Epstein J, Santo RM, Guillemin F. A review of guidelines for cross-cultural adaptation of questionnaires could not bring out a consensus. J Clin Epidemiol. 2015;68(4):435-41.

25. Bijl JV, Poelgeest-Eeltink AV, Shortridge-Baggett L. The psychometric properties of the diabetes management self-efficacy scale for patients with type 2 diabetes mellitus. J Adv Nurs. 1999:30(2):352-9.

26. Sharoni SK, Wu SF. Self-efficacy and self-care behavior of Malaysian patients with type 2 diabetes: a cross sectional survey. Nurs Health Sci. 2012;14(1):38-45.

27. Vivienne Wu SF, Courtney M, Edwards H, McDowell J, Shortridge-Baggett LM, Chang PJ. Development and validation of the Chinese version of the diabetes management self-efficacy scale. Int J Nurs Stud. 2008;45(4):534-42.

28. Bandura A. Social cognitive theory of self-regulation. Organ Behav Hum Decis Process. 1991;50(2):248-87.

29. Cameron L, Leventhal $H$. The self-regulation of health and illness behaviour. London: Routledge; 2003.

30. Skevington SM, Lotfy M, O'Connell KA. The World Health Organization's WHOQOL-BREF quality of life assessment: psychometric properties and results of the international field trial. A report from the WHOQOL group. Qual Life Res. 2004;13(2):299-310.

31. Development of the World Health Organization WHOQOL-BREF quality of life assessment. The WHOQOL group. Psychol Med. 1998;28(3):551-8.

32. Hasanah Cl, Naing L, Rahman AR. World Health Organization quality of life assessment: brief version in Bahasa Malaysia. Med J Malaysia. 2003;58(1):79-88.

33. Al-Qazaz H, Hassali MA, Shafie AA, Sulaiman SA, Sundram S, Morisky DE. The eight-item Morisky medication adherence scale MMAS: translation and validation of the Malaysian version. Diabetes Res Clin Pract. 2010;90(2):216-21.

34. Morisky DE, Green LW, Levine DM. Concurrent and predictive validity of a self-reported measure of medication adherence. Med Care. 1986;24(1):67-74.

35. Krousel-Wood M, Islam T, Webber LS, Re RN, Morisky DE, Muntner P. New medication adherence scale versus pharmacy fill rates in seniors with hypertension. Am J Manag Care. 2009;15(1):59-66.

36. Morisky DE, DiMatteo MR. Improving the measurement of self-reported medication nonadherence: response to authors. J Clin Epidemiol. 2011 64(3):255-7. discussion 258-263

37. Kucukarslan SN. A review of published studies of patients' illness perceptions and medication adherence: lessons learned and future directions. Res Social Adm Pharm. 2012;8(5):371-82.

38. Horne R, Chapman SC, Parham R, Freemantle N, Forbes A, Cooper V. Understanding patients' adherence-related beliefs about medicines prescribed for long-term conditions: a meta-analytic review of the necessity-concerns framework. PLoS One. 2013;8(12):e80633.

39. Broadbent E, Donkin L, Stroh JC. Illness and treatment perceptions are associated with adherence to medications, diet, and exercise in diabetic patients. Diabetes Care. 2011;34(2):338-40.

40. Sweileh WM, Zyoud SH, Abu Nab'a RJ, Deleq MI, Enaia MI, Nassar SM, Al-Jabi SW. Influence of patients' disease knowledge and beliefs about medicines on medication adherence: findings from a cross-sectional survey among patients with type 2 diabetes mellitus in Palestine. BMC Public Health. 2014;14:94.

41. Fisher L, Hessler DM, Polonsky WH, Mullan J. When is diabetes distress clinically meaningful?: establishing cut points for the diabetes distress scale. Diabetes Care. 2012;35(2):259-64.

42. Chew BH, Mukhtar F, Sherina MS, Paimin F, Hassan NH, Jamaludin N. The reliability and validity of the Malay version 17-item diabetes distress scale. Malays Fam Physician. 2015;10(2):22-35.

43. Ogbera A, Adeyemi-Doro A. Emotional distress is associated with poor self care in type 2 diabetes mellitus. J Diabetes. 2011;3(4):348-52.

44. Fisher L, Mullan JT, Arean P, Glasgow RE, Hessler D, Masharani U. Diabetes distress but not clinical depression or depressive symptoms is associated with glycemic control in both cross-sectional and longitudinal analyses. Diabetes Care. 2010;33(1):23-8.

45. Aikens JE. Prospective associations between emotional distress and poor outcomes in type 2 diabetes. Diabetes Care. 2012;35(12):2472-8.
46. Bridges HA, Smith MA. Mediation by illness perceptions of the association between the doctor-patient relationship and diabetes-related distress. J Health Psychol. 2016;21(9):1956-65.

47. Sherina MS, Arroll B, Goodyear-Smith F. Criterion validity of the PHQ-9 (Malay version) in a primary care clinic in Malaysia. Med J Malaysia. 2012;67(3):309-15.

48. Kroenke K, Spitzer RL, Williams JB. The PHQ-9: validity of a brief depression severity measure. J Gen Intern Med. 2001;16(9):606-13.

49. Fayers $P$, Machin D. Quality of life: the assessment, analysis and interpretation of patient-reportedoutcomes. 2nd ed. West Sussex, England: Wiley; 2013.

50. Burns RJ, Deschenes SS, Schmitz N. Cyclical relationship between depressive symptoms and diabetes distress in people with Type 2 diabetes mellitus: results from the Montreal evaluation of diabetes treatment cohort study. Diabet Med. 2015;32:1272-8.

51. Mc Sharry J, Moss-Morris R, Kendrick T. Illness perceptions and glycaemic control in diabetes: a systematic review with meta-analysis. Diabet Med. 2011;28(11):1300-10

52. Chew BH, Vos RC, Sazlina SG, Shamsuddin NH, Fernandez A, Mukhtar F, Mastura I, Mohd Ahad A, Sundram NN, Ali SZ, et al. The effectiveness of a value-based EMOtion-cognition-focused educatlonal programme to reduce diabetes-related distress in Malay adults with type 2 diabetes (VEMOFIT): study protocol for a cluster randomised controlled trial. BMC Endocr Disord. 2017;17(1):22.

53. Fisher L, Gonzalez JS, Polonsky WH. The confusing tale of depression and distress in patients with diabetes: a call for greater clarity and precision. Diabet Med. 2014;31(7):764-72.

54. Wilson RS, Schneider JA, Boyle PA, Arnold SE, Tang Y, Bennett DA. Chronic distress and incidence of mild cognitive impairment. Neurology. 2007; 68(24):2085-92.

55. Lin YP, Chiu KM, Wang TJ. Reliability and validity of the Chinese version of the brief illness perception questionnaire for patients with coronary heart disease. Journal of Oriental Institute of Technology. 2011;31:145-56.

56. Kossakowska MM, Stefaniak TJ. Psychometric properties for the polish version of the brief illness perception questionnaire (brief IPQ). Health Psychology Report. 2017;5(1):67-83.

57. de Raaij EJ, Schroder C, Maissan FJ, Pool JJ, Wittink H. Cross-cultural adaptation and measurement properties of the brief illness perception questionnaireDutch language version. Man Ther. 2012;17(4):330-5.

58. Mokkink LB, Terwee CB, Knol DL, Stratford PW, Alonso J, Patrick DL, Bouter LM, de Vet HC. The COSMIN checklist for evaluating the methodological quality of studies on measurement properties: a clarification of its content. BMC Med Res Methodol. 2010;10:22.

\section{Submit your next manuscript to BioMed Central and we will help you at every step:}

- We accept pre-submission inquiries

- Our selector tool helps you to find the most relevant journal

- We provide round the clock customer support

- Convenient online submission

- Thorough peer review

- Inclusion in PubMed and all major indexing services

- Maximum visibility for your research

Submit your manuscript at www.biomedcentral.com/submit 